\title{
Low seroprevalence of Coxiella burnetii in Boer goats in Missouri
}

\author{
Molly D Baker and Patrick O Pithua*
}

\begin{abstract}
Background: Goats are known reservoirs of Coxiella burnetii, the etiologic agent of Q fever. However, there has been very little research on the prevalence of $C$. burnetii exposure and risk in meat goats farmed in the US. Banked serum samples were secondarily tested for $C$. burnetii specific antibodies.

Findings: The animal and herd-level seroprevalence estimates for C. burnetii were 1.2\% (3/249) and 4.2\% (1/24) respectively. Within-herd seroprevalence ranged from $0 \%$ to $1.2 \%$.

Conclusions: This study indicates that seroprevalence of $C$. burnetii in Boer goats raised in Missouri was low, but it does not preclude the existence of a higher level of infection in Missouri's meat goat herds. This result is inconclusive because this study was disadvantaged by the small number of individual animal and herds tested, which compromised the statistical power of this study to detect a possible higher seroprevalence of $C$. burnetii in this population, if present. More research is warranted to corroborate the preliminary findings reported here in order to determine the public health significance C. burnetii infection risks associated with contemporary goat production systems in the US.
\end{abstract}

Keywords: Coxiella burnetii, Goats, Q fever, Missouri, United States

\section{Findings \\ Background}

Coxiella burnetii, an obligate intracellular pathogen of both humans and animals is the causative agent of Qfever [1]. Zoonotic Q fever in humans has generally been linked with transitory outbreaks of the disease in animals $[2,3]$. Humans are primarily infected through inhalation of $C$. burnetii in aerosols, resulting from occupational exposures, although transmissions through oral ingestion of contaminated food are documented [4,5]. While infected ruminants remain largely asymptomatic, the primary signs of $C$. burnetii include spontaneous, late-term abortions in pregnant animals. There is evidence that $C$. burnetii is a public health hazard in the US with humans being exposed to the bacteria through milk. A recent report found that $94 \%$ of bulk tank milk samples collected from US dairy herds contained C. burnetii specific DNA [6]. Loftis and others detected C. burnetii in 42.9\% (9/21) of commercial raw milk samples in the US [7], and a recent case

\footnotetext{
*Correspondence: pithuap@missouri.edu

Department of Veterinary Medicine \& Surgery, Veterinary Medicine Teaching Hospital, College of Veterinary Medicine, University of Missouri, $900 \mathrm{E}$. Campus Drive, Columbia, MO 65211, USA
}

report found Q fever clusters among raw milk consumers in the State of Michigan [4].

Small ruminants and goats, in particular, are primary reservoirs of C. burnetii [8]. A review of the burden of Q fever in the US revealed a $41.6 \%$ average prevalence of $C$. burnetii infection in goats [9]. Studies in sheep in Wyoming revealed a 7\% seroprevalence of C. burnetii [10]. Recent Studies in California [9,11] and Washington [12] suggested that C. burnetii was endemic in US meat and milk goats, although its prevalence may be underestimated and the overall distribution of the disease in the US remains unknown $[9,11,12]$. No studies have evaluated the prevalence and risk factors of C. burnetii infections in meat goats farmed in Missouri. The aim of this pilot investigation was to determine whether Boer goats, the preponderant meat goat breed in Missouri, were exposed to C. burnetii and to estimate seroprevalence of the exposure.

\section{Methods}

There are approximately 3,955 goat herds in Missouri with an estimated 103,669 goats [13]. Based on the 2012 agricultural census data, Boer goats are a dominant meat breed accounting for over $86 \%$ of all goats in Missouri 
[13]. In this study, banked serum samples from a previous study performed to estimate the apparent prevalence of Mycobacterium avium subsp. paratuberculosis (MAP) in Missouri Boer goat herds were secondarily tested for C. burnetii specific antibodies [14]. Participation in the original study was voluntary and protocols were approved by the Institutional Animal Use and Care Committee at the University of Missouri (Protocol \# 7395). Herds and animals were selected based on criteria described elsewhere [14]. Briefly, Boer goat herds in the State of Missouri constituted the sampling unit, and the target population included goats 24 months of age and older. Herds containing other breeds of goats were excluded. Sixty-one herds were required to estimate seroprevalence of at least $20 \%$ for MAP, with an allowable error of $10 \%$ and a $5 \%$ type I error rate. Twenty-five (41\%) herds of the required 61 herds were ultimately enrolled due to low farmer response rates.

Herd visits for sample collection were completed between May and September, 2012, and all eligible animals (i.e. Boer goats $\geq 24$ months old) present in herds that agreed to the study were tested. The mean \pm SD (min., max.) number of animals sampled per herd was $25 \pm 19$ $(2,57)[14]$. The number of herds sampled that had $<50$, 50-100, and 100-200 goats were 19 (76\%), 5 (20\%), and 1 (4\%) respectively [14].

Blood samples were collected via jugular venepuncture from a total of 629 goats, centrifuged at $3,000 \mathrm{~g}$ and the resulting sera stored at $-80^{\circ} \mathrm{C}$. Only a subset of these banked serum samples was randomly selected and tested for $C$. burnetii due to budgetary constraints. Of the 629 sera available, 249 were submitted to the National Veterinary Services Laboratories in Ames, Iowa, for processing and testing. These samples were tested for C. burnetii antibodies using a commercial ELISA kit following the manufacturer's guidelines (IDEXX Switzerland AG, Liebelfld-Bern, Switzerland). The kit consisted of micro titre plate wells laced with phase I and II inactivated C. burnetii antigens from the Nile Mile strain on which Q fever specific antibodies bind [15]. Plates were washed to remove unbound antibodies. A peroxidaselabeled anti-ruminant immunoglobulin $\mathrm{G}$ was added to the $C$. burnetii antigen-antibody complex in each well. The micro titre wells were incubated and then washed to remove unbound reagents. The optical density (OD) value of each sample was measured by a spectrophotometer (CHEKIT Q Fever Antibody ELISA, IDEXX Switzerland AG, Liebelfld-Bern, Switzerland). Seropositivity was determined as a percentage of test samples' OD value relative to the positive control, and corrected for the OD values from the negative control. Test samples with $\mathrm{OD}$ values $<30 \%$ were considered negative, samples with OD values between $30 \%$ and $40 \%$ were reported as suspect, and those with OD values $\geq 40 \%$ were declared positive for Q-fever antibodies. The animal level, within-herd, and between-herd seroprevalence were calculated by dividing the number of test positive outcomes by the appropriate denominator for each measure. Confidence intervals (95\%) for the estimated seroprevalence were calculated using the Wilson binomial approximation method as described [16].

\section{Results and discussion}

In total, 249 serum samples were tested. Ninety-eight percent $(244 / 249)$ of these were negative (OD <30\%), $0.8 \%$ $(2 / 249)$ were suspects $(\geq 30 \%$ OD $\leq 40 \%)$, and $1.2 \%(3 / 249)$ were positive for $C$. Burnetii antibodies. Based on test results, animal-level, herd-level and within-herd (range) seroprevalence estimates were $1.2 \%$ (95\% CI $=0.4 \%-3.5 \%)$, $4.2 \%(95 \%=0.7 \%-20.2 \%)$, and $0 \%$ to $1.2 \%$, respectively. This estimate was lower than previously reported in California dairy goats (i.e. 24\%) [11]. Similarly, the apparent prevalence estimates in this study were lower than the $8 \%$ (144/1794) animal-level; 8.6\% (9/105) herd-level; and 2.9\% to $75.8 \%$ within-herd level estimates for C. burnetii seroprevalence reported in a recent study of domestic goats in Washington [12].

In the current study, only Boer goats (i.e., a meat breed) were tested. In contrast, the Washington study focused on goats of all production types (i.e. meat and dairy goats) while only dairy goats were studied in California $[11,12]$. Thus, the observed disparity in C. burnetii seroprevalence between the current study and those performed in Washington, and California was likely a function of breed (or production types) predisposition to $C$. burnetii infection with apparent risks being greater for dairy breeds of goats than meat breeds (i.e. Boer goats). However, while the most-recent large-scale C. burnetii outbreaks in humans in The Netherlands were linked to dairy goat farms as the source [3], no plausible explanation exists in support of the breed-susceptibility hypothesis. Increased levels of exposure, due to a high within-herd C. burnetii prevalence rather than genetic or breed predisposition, is a more likely reason for the apparently higher prevalence of infection in the dairy breeds seen in California [11]. The low C. burnetii seroprevalence observed in this study could also be due to differences in the mobility patterns of dairy versus meat goats. For instance, in dairy goat management, goats may be moved to other farms over their lifetime as owners' buy-in or sell-out animals as herd replacements [2]. Between-herds mobility is a well-recognized risk factor for infectious disease transmission in livestock and is likely a more common phenomenon in dairy versus meat goats hence the higher animal and herd seroprevalence of C. burnetii observed in dairy relative to the meat breeds of goats [2].

It is possible that the higher prevalence of $C$. burnetii observed in dairy goat breeds may be the consequences 
of differences in management between the two production types. Goats for meat in the US are raised mainly under an extensive or grazing system [17]. Infectious disease such as C. burnetii likely spreads faster under a confinement system of production. The latter is typical for most dairy goats in the US.

The current study was similar to the Washington survey in the use of an ELISA with phase I and II antigens from the Nile Mile strain of $C$. burnetii to detect antibodies in sera $[12,15]$. Moreover, in both studies only goats from herds with no known history of C. burnetii infections were tested. However, the difference in the $C$. burnetii seroprevalence observed between Missouri, and California may have been due to the difference in diagnostic tests used, given that sera from dairy goats in California were tested using a micro agglutination assay containing phase II antigens from the C76 C. burnetii strain [11].

Though a recent outbreak of $\mathrm{Q}$ fever in humans was linked to a single dairy goat farm in Washington State [2], underscoring the significance of goat associated zoonotic risk of $C$. burnetii in the US, to date, risk factors for the emergence of C. burnetii in US goat populations have not been fully studied. A recent study in The Netherlands found an association between large flock size (i.e., $\geq 800$ goats), proximity to infected herds (i.e., $<8 \mathrm{Km}$ ), high cattle density in the flock district $\left(\geq 800\right.$ cattle $\left./ \mathrm{Km}^{2}\right)$, presence of cats or dogs in the goat pens, and the use of imported straw, and C. burnetii seroprevalence in commercial dairy goat farms [18]. However, the epidemiology of C. burnetii infections in goats in The Netherlands may not reflect the epidemiology of the disease in the US caprine population given differences in goat productions systems. To better understand the human health risk of $C$. burnetii infection associated with contemporary goat production systems in the US, and to develop appropriate interventions to limit occupational exposure and public health risks, knowledge of prevalence, distribution, and risk factors for C. burnetii infections in meat goats are required.

A disadvantage of this study was that it was impossible to ascertain the counties and therefore, the geographic scope of the disease in Missouri represented in the samples tested because of their secondary nature. Additionally, the relatively few goats tested in this study may have undermined the statistical power of this study to detect a larger number of exposed animals hence the low seroprevalence observed. It entirely is possible that the seroprevalence of $C$. burnetii in Boer goats in Missouri are higher than could be demonstrated with the current data. The results reported here is inconclusive. We contend that if the animal and herd level apparent prevalence of C. burnetii in Boer goats in Missouri are as high as reported in California or Washington [11,12], then future studies in Missouri will require a state-wide representative sample of $\geq 984$ goats from at least 453 herds to estimate an animal-level seroprevalence $\geq 20 \%$ or a herd-level apparent prevalence $\geq 8 \%$ with $95 \%$ confidence level, assuming an infinite population of goats in Missouri.

\section{Conclusions}

To our knowledge, this is the first study of C. burnetii seroprevalence in Missouri Boer goat herds. While this study found a low seroprevalence of $C$. burnetii in a limited sample of Boer goats from Missouri, the results do not preclude the existence of infection in Missouri's Boer goat herds. The results shown here is likely indicative of a naïve population of goats, which if sufficiently exposed to animals actively shedding C. burnetii, could acquire prevalence as high if not higher than previously reported in the US $[9,11,12]$. This study was limited by the small number of individual animal and herds tested. This may have compromised the statistical power of this study to detect a possible higher seroprevalence. There is a need for largescale surveillance studies to determine the underlying risk factors for emergence and transmission of $C$. burnetii in this rapidly growing US livestock sector.

\section{Competing interests}

The authors declare that they have no competing interests.

\section{Authors' contributions}

$M D B$, a summer research scholar, participated in the design of the study and performed the statistical analysis. PP conceived of the study, provided the samples, and participated in its design and coordination and helped to draft the manuscript. All authors read and approved the final manuscript.

\section{Acknowledgements}

This research was supported partially by a Committee on Research Grant from the College of Veterinary Medicine, University of Missouri-Columbia.

Received: 31 December 2013 Accepted: 30 June 2014

Published: 4 July 2014

\section{References}

1. Karakousis PC, Trucksis M, Dumler JS: Chronic Q Fever in the United States. J Clin Microbiol 2006, 44(6):2283-2287.

2. Centers for Disease Control and Prevention (CDC): Notes from the field: $\mathbf{Q}$ fever outbreak associated with goat farms-Washington and Montana, 2011. MMWR Morb Mortal Wkly Rep 2011, 60(40):1393.

3. van de Wijngaard CC, Dijkstra F, van PELT W, van Asten L, Kretzschmar M, Schimmer B, Nagelkerke NJD, Vellema P, Donker GA, Koopmans MPG: In search of hidden Q-fever outbreaks: linking syndromic hospital clusters to infected goat farms. Epidemiol Infect 2011, 139(01):19-26.

4. Signs KA, Stobierski MG, Gandhi TN: Q fever cluster among raw milk drinkers in Michigan, 2011. Clin Infect Dis 2012, 55(10):1387-1389.

5. Walsh MG: Assessing Q fever in a representative sample from the United States population: identification of a potential occupational hazard. Epidemiol Infect 2012, 140(1):42-46.

6. Kim SG, Kim EH, Lafferty CJ, Dubovi E: Coxiella burnetii in bulk tank milk samples, United States. Emerg Infect Dis 2005, 11(4):619-621.

7. Loftis AD, Priestley RA, Massung RF: Detection of Coxiella burnetii in commercially available raw milk from the United States. Foodborne Pathog Dis 2010, 7(12):1453-1456.

8. Eibach R, Bothe F, Runge M, Fischer SF, Philipp W, Ganter M: Q fever: baseline monitoring of a sheep and a goat flock associated with human infections. Epidemiol Infect 2012, 140(11):1939-1949.

9. McQuiston $\mathrm{JH}$, Childs JE: Q fever in humans and animals in the United States. Vector Borne Zoonotic Dis 2002, 2(3):179-191. 
10. Loftis AD, Reeves WK, Miller MM, Massung RF: Coxiella burnetii, the agent of $\mathrm{Q}$ fever, in domestic sheep flocks from Wyoming, United States. Vector Borne Zoonotic Dis 2012, 12(3):189-191.

11. Ruppanner R, Riemann HP, Farver TB, West G, Behymer DE, Wijayasinghe C: Prevalence of Coxiella burnetii ( $Q$ fever) and Toxoplasma gondii among dairy goats in California. Am J Vet Res 1978, 39(5):867-870.

12. Sondgeroth KS, Davis MA, Schlee SL, Allen AJ, Evermann JF, McElwain TF, Baszler TV: Seroprevalence of Coxiella burnetii in Washington State domestic goat herds. Vector Borne Zoonotic Dis 2013, 13(11):779-783.

13. Goats, Kids, and Mohair - Inventory, Mohair Production, and Sales: 2012 and 2007. [http://www.agcensus.usda.gov/Publications/2012/Full_Report/ Volume_1,_Chapter_1_State_Level/Missouri/st29_1_028_031.pdf]

14. Pithua P, Kollias NS: Estimated prevalence of caprine paratuberculosis in boer goat herds in missouri. USA Vet Med Int 2012, 2012:674085.

15. Emery MP, Ostlund EN, Schmitt BJ: Comparison of Q fever serology methods in cattle, goats, and sheep. J Vet Diagn Invest 2012, 24(2):379-382.

16. Brown LD, Cat TT, Gupta D: Interval Estimation for a proportion. Stat Sci 2001, 16:101-133.

17. Sahlu T, Goetsch AL: A foresight on goat research. Small Ruminant Res 2005, 60(1-2):7-12.

18. Schimmer B, Luttikholt S, Hautvast J, Graat E, Vellema P, van Duynhoven Y: Seroprevalence and risk factors of $\mathrm{Q}$ fever in goats on commercial dairy goat farms in the Netherlands, 2009-2010. BMC Vet Res 2011, 7(1):81.

doi:10.1186/1756-0500-7-421

Cite this article as: Baker and Pithua: Low seroprevalence of Coxiella

burnetii in Boer goats in Missouri. BMC Research Notes 2014 7:421.

\section{Submit your next manuscript to BioMed Central and take full advantage of:}

- Convenient online submission

- Thorough peer review

- No space constraints or color figure charges

- Immediate publication on acceptance

- Inclusion in PubMed, CAS, Scopus and Google Scholar

- Research which is freely available for redistribution 